\title{
Development, feasibility and acceptability of an intervention to improve care for agitation in people living in nursing homes with dementia nearing the end-of-life
}

Professor Elizabeth L Sampson ${ }^{1 *}$, Dr Julie Barber ${ }^{2}$, Juliet Gillam ${ }^{3}$, Dr Francesca La Frenais ${ }^{3}$, Katie Lambe ${ }^{3}$, Dr Anne Laybourne ${ }^{3}$, Dr Monica Manela ${ }^{4}$, Dr Louise Marston ${ }^{4}$, Dr Kirsten Moore ${ }^{1}$, Monica Panca ${ }^{4}$, Aisling Stringer ${ }^{3}$, Lucy Webster ${ }^{3}$, Professor Gill Livingston ${ }^{3}$

${ }^{1}$ Marie Curie Palliative Care Research Unit, Division of Psychiatry, University College London, Maple House, 149 Tottenham Court Road, London, W1T 7NF, UK

2 Department of Statistical Science and Priment Clinical Trials Unit, University College, London WC1E 6BT

${ }^{3}$ Department of Old Age Psychiatry, Division of Psychiatry, University College London, Maple House, 149 Tottenham Court Road, London, W1T 7NF, UK

${ }^{4}$ Department of Primary Care and Population Health and Priment Clinical Trials Unit, University College London, Rowland Hill Street, London, NW3 2PF, UK.

\section{${ }^{*}$ Corresponding author}

E-mail: e.sampson@ucl.ac.uk

Fax: 02076799426

Telephone: 02076799730

Postal Address: $6^{\text {th }}$ Floor, Maple House, 149 Tottenham Court Road, London, W1T 7NF, UK

Word count: 4990 


\section{ABSTRACT}

Objectives: To develop a staff training intervention for agitation in people with severe dementia, reaching end-of-life, residing in nursing homes (NHs), test feasibility, acceptability and whether a trial is warranted.

Design: Feasibility study with pre- and post-intervention data collection, qualitative interviews and focus groups.

Setting: Three NHs in south-east England with dementia units, diverse in terms of size, ownership status and location.

Participants: Residents with a dementia diagnosis or scoring $\geq 2$ on the Noticeable Problems Checklist, rated as "severe" on Clinical Dementia Rating Scale, family carers and staff (healthcare assistants and nurses).

Intervention: Manualised training, delivered by non-clinical psychology graduates focussing on agitation in severe dementia, underpinned by a palliative care framework.

Measurements: Main outcomes were feasibility of recruitment, data collection, follow-up and intervention acceptability. We collected resident, family carer and staff demographics. Staff provided data on resident's agitation, pain, quality of life and service receipt. Staff reported their sense of competence in dementia care. Family carers reported on satisfaction with end-of-life care. In qualitative interviews, we explored staff and family carers' views on the intervention.

Results: The target three NHs participated: 28 (49\%) residents, 53 (74\%) staff and 11 (85\%) family carers who were eligible to participate consented. $84 \%$ of staff attended $\geq 3$ sessions, and we achieved $93 \%$ follow-up. We were able to complete quantitative interviews. Staff and family carers reported the intervention and delivery were acceptable and helpful.

Conclusions: The intervention was feasible and acceptable indicating a larger trial for effectiveness may be warranted.

Keywords: dementia, palliative care, nursing home, feasibility study, agitation 
Improving care for agitation in dementia at end-of-life

\section{INTRODUCTION}

Worldwide, 47 million people are living with dementia and because of increasing longevity this is expected to triple by 2050 (Prince et al., 2015). Dementia is now the third commonest cause of death in higher income countries (World Health Organisation, 2017), and one third of the United Kingdom (UK) population aged over 65 will die with dementia (Xie et al., 2008). Most people with dementia in the UK will die in a care home (Sleeman et al., 2016) and require a palliative care approach, underpinned by careful assessment of symptoms and a holistic approach to care (World Health Organisation, 1990) (van der Steen et al., 2014).

In the UK more than 420,000 people aged over 65 years live in residential care, of which approximately 220,000 reside in care homes with nursing (referred to henceforth as "nursing homes" (NHs)) (Laing and Buisson, 2017). Staff working in NHs face considerable challenges. They may have few qualifications, are poorly paid, and may be migrant workers (Skills for Care, 2019). There are recruitment challenges and high staff turnover (Orellana, 2014). Interventions developed to improve the quality of end-of-life care received by $\mathrm{NH}$ residents often require high levels of external facilitation (Froggatt et al., 2006) and skilled (and thus costly) staff for implementation (Moore et al., 2017a). These may not be feasible, cost-effective or scalable in the UK NH sector. In addition, most interventions address a range of problems, rather than focussing on specific issues such as agitation (Smets et al., 2018).

Agitation is one of the most common neuropsychiatric symptoms of dementia and includes restlessness, pacing, shouting and verbal or physical aggression. It occurs in over $50 \%$ of $\mathrm{NH}$ residents (Livingston et al., 2017) and may be related to neurodegeneration or unmet needs such as pain, boredom, hunger or thirst. Agitation and distress are very common towards end-of-life, when people with severe dementia may struggle to communicate their needs, affect $75 \%$ of people with dementia, and persist even in the last month of life (Aminoff and Adunsky, 2006; Mitchell et al., 2009). Essential components of palliative care such as adequate pain management and attention to psychosocial and spiritual factors are often neglected (Sampson et al., 2018). Interventional research on providing palliative care for people with dementia is scant and has been criticised for lacking a strong theoretical basis (van der Steen and Goodman, 2015).

\section{Underpinning theory for intervention development}

This study is part of the Managing Agitation and Improving QUality of lifE (MARQUE) programme, which aimed to better understand causes of agitation across care settings and 
develop interventions to improve care. Work was underpinned by a conceptual framework viewing agitation as usually being a manifestation of unmet needs, which we have demonstrated are strongly linked to lower quality of life (QoL) (Livingston et al., 2017; Livingston et al., 2014a). Needs can be unmet because the person with dementia has difficulties in knowing, or making these known. Unmet needs encompass enjoyable activities, stimulation (Rapaport et al., 2018), comfort, pain relief (Husebo et al., 2011) or physical contact. External factors include the degree to which staff are available, aware and skilled in providing activities, physical and emotional comfort (Livingston et al., 2014b). Further theoretical development highlighted the importance of staffs' own sense of valued personhood (Kadri et al., 2018; Sampson et al., 2019).

To develop our intervention, we refined our theory in the context of end-of-life. We conducted non-participant observations (observing care without actively participating) in $\mathrm{NHs}$ and hospitals of people with severe dementia or those with moderate dementia with acute physical illness or chronic comorbidity, which may indicate they were near end-of-life. Some staff saw agitation as a signal of need, others felt it was purposeless behaviour; their reactions included fear and a sense of "not knowing what to do" (Sampson et al., 2019). Staff who saw agitation as purposeless often withdrew, focussing on tasks and avoiding social interaction or identifying and managing the cause of agitation. This increased loss of personhood, reduced interaction and further worsened agitation. This theory informed a staff training intervention to improve care for agitation in people with severe dementia residing in $\mathrm{NHs}$.

\section{AIM}

To test intervention feasibility and acceptability to inform whether a randomised controlled trial is indicated. Specific objectives were to:

1) Describe recruitment and consent rates for $\mathrm{NHs}$, residents, family carers and staff to inform a larger trial

2) Assess feasibility of intervention delivery as whether at least $60 \%$ of staff attend at least three of the four sessions

3) Assess whether the intervention was acceptable

4) Assess the feasibility of collecting measures of QoL and use of health and social care resources

5) Explore potential outcome measures for a further study

6) Estimate the costs of delivering the intervention and associated health and social care costs 


\section{METHODS}

\section{Training development}

The study team, including experts in dementia care, palliative care and $\mathrm{NH}$ staff drafted the manuals, based on theory described above, with topics informed by longitudinal data on unmet needs in $\mathrm{NH}$ residents with severe dementia and their family carers (Moore et al., 2017b; Sampson et al., 2018). Each topic was framed in the holistic palliative care model encompassing physical, psychological, social and spiritual domains (Clark, 1999). The four session themes were: 1) understanding severe dementia, 2) pain and discomfort in severe dementia, 3) working with others during the severe stages of dementia and 4) what works? Using new skills and strategies for the future. Our prior work and theory highlighted how neglect of staff personhood leads to burnout and emotional exhaustion. We therefore supported staff to reflect on their emotional response to caring for people with severe dementia who were agitated. Sessions included reflection on experiences, a recap of previous session(s), validation of existing knowledge and expertise, interactive group tasks, talking points and ended with a relaxation exercise (figure 1).

Figure 1

\section{Training delivery}

We designed the intervention to be sustainable and facilitated by two supervised non-clinical psychology graduates, trained to deliver the intervention. We piloted manuals with five $\mathrm{NH}$ staff amending them to improve clarity and delivery style. We wrote a companion facilitator manual for each session. We delivered sessions between two and four times in each $\mathrm{NH}$ so all eligible staff could attend.

\section{Study type and setting}

We conducted a non-randomised feasibility study in three NHs. We planned to recruit three $\mathrm{NHs}$ with distinct dementia units, sampled for diversity in terms of size, ownership status and location. We required them to commit to the training being compulsory for all day staff. We excluded homes due to close in the next 12 months or located more than 60 minutes' travel from the study base. 


\section{Ethics and consent procedures}

The London - Queen Square Research Ethics Committee (17/LO/1400) approved the study. $\mathrm{NH}$ managers gave consent for their $\mathrm{NH}$ to participate. Potential resident participants had severe memory problems and did not have capacity to consent for themselves. Using the Mental Capacity Act (2005) we used a key family member or friend as a "personal consultee" and obtained written assent for the resident. For the observation of the quality of staffresident interactions, we obtained collective consent from the $\mathrm{NH}$ manager. The $\mathrm{NH}$ manager informed staff, who were given information sheets, that they could opt out of being observed at any time and the researcher would cease observations. We approached individual NH staff via their manager and they gave individual signed informed consent to participate in the focus groups. Family carers of residents recruited to the study were invited to participate and gave signed informed consent for this.

\section{Study population}

Residents had a diagnosis of dementia or a score $\geq 2$ on the Noticeable Problems Checklist which has been validated against clinical diagnosis (Levin et al., 1989; Moriarty and Webb, 2000) and were rated "3-severe" on the Clinical Dementia Rating Scale (CDR) (Morris, 1993). Eligible staff were; permanently employed healthcare assistants, activity coordinators and qualified nurses who worked at least some day shifts (enabling them to attend training) on the NH's dementia units. We aimed to recruit $60 \%$ of all eligible staff.

\section{Intervention implementation}

The study ran in three NHs over 14 weeks (pre-intervention data collection weeks 1-6, intervention period weeks 7-10, follow-up during weeks 11-14). We invited staff to attend the four sessions, which lasted two hours each, including a break. In addition, we offered catch up sessions at each $\mathrm{NH}$ for those who were ill or on leave. We documented the number of group intervention sessions delivered, number of staff at each session, the proportion of staff who attended at least three sessions and who attended additional catch-up sessions. We explored intervention feasibility and acceptability with staff and family carers using semistructured qualitative interviews. We asked what they thought of manual layout, content and practical tasks. We asked staff how effectively facilitators managed group conversations, whether the programme was sustainable and deliverable by non-professional facilitators. 


\section{Data collection}

Data were collected by research assistants prior to intervention (weeks 1-6) and at follow-up after intervention delivery (weeks 11-14).

\section{Resident data}

At baseline, we collected demographic data. At baseline and follow-up, we assessed residents via proxy interviews with staff using:

- Cohen Mansfield Agitation Inventory (CMAI). Scores >45 indicate clinically significant agitation (Cohen-Mansfield et al., 1989).

- Pain Assessment in Advanced Dementia (PAINAD) (Warden et al., 2003). To assess pain during a care task and at rest. Scores $\geq 2$ indicate pain.

- Symptom Management at the End-of-Life in Dementia (SMEOLD), range 0-45, higher scores indicating greater comfort (Kiely et al., 2006).

- If a resident died during the study we aimed to rate comfort around the time of death with the Comfort Assessment in Dying with Dementia (CAD-EOLD) (range 14-42, higher scores indicating better comfort when dying) (Kiely et al., 2006).

- DEMQOL-Proxy (range 31-124, higher score indicates better QoL) (Smith et al., 2007). We also used the Quality of Life in Late Stage Dementia (QUALID) scale (range 11-55 lower scores indicate better QoL) (Weiner et al., 2000), and EuroQol EQ-5D 5 (EQ-5D5L- Proxy) (Rabin and de, 2001) with the associated UK tariff (Devlin et al., 2017; Herdman et al., 2011).

- The modified Client Service Receipt Inventory (CSRI) recorded health and social care resource use from resident files and NH staff (Beecham and Knapp, 2001).

\section{Family carer data}

Prior to intervention, we collected data on age, sex, relationship to resident and number of visits per month. Prior to intervention and at follow-up, we assessed family carer satisfaction using the Satisfaction with Care at the End-of-Life in Dementia Scale (SWCEOLD) which has 10 items, score range 10-40 with higher scores indicating greater satisfaction (Kiely et al., 2006)

\section{Staff data}

We collected staff demographic data at baseline; sex, ethnicity, highest level of educational 
attainment, English as a first language, years working in the $\mathrm{NH}$ and the sector, working pattern and whether they held a UK-recognised nursing qualification. Prior to intervention and at follow-up they completed the Sense of Competence In Dementia Scale (SCID) (Schepers et al., 2012) a self-report of staffs' subjective competence, with four subscales (professionalism, building relationships, care challenges, sustaining personhood, range 1768 , higher scores indicate greater competence).

\section{Nursing home data}

We collected data from $\mathrm{NHs}$ on management and ownership (private or charity), CQC registration type (dementia registered, dementia specialist, mental health registered, physical disability registration), number of beds, CQC rating domains (safe, effective, caring, responsive, well led and overall), whether or not there was a specialist unit for residents with behavioural issues. We documented numbers of staff rostered during days and nights, agency staff and permanently registered staff, the number of residents and staff dementia training in the previous 6 months.

\section{Staff-resident interactions}

We collected data at baseline and follow-up on the quality of interactions between residents and staff using the Quality of Interactions Schedule (QUIS), an observational tool rating staffresident interactions on an ordinal scale as 'positive' (2), 'neutral' (1), or 'negative' (0) (Dean et al., 1993). The QUIS has good inter-rater reliability (Dean et al., 1993) and reasonable test-retest reliability (McLean et al., 2017).

\section{Qualitative interviews}

We held focus groups with a variety of $\mathrm{NH}$ staff to explore their views on the training programme, manuals, facilitators and barriers to the implementation of the training and triangulate data. We showed family carers the manuals and gave an overview of the training programme prior to their interviews. These were audiotaped and transcribed verbatim and entered onto a qualitative software programme (Nvivo) for coding, management and retrieval of data.

\section{Sample size}

No formal power calculation was carried out for this feasibility and acceptability study. Informed by our previous $\mathrm{NH}$ research (Livingston, 2019), we aimed to recruit three $\underline{\mathrm{NHs}}$ which we expected to include approximately 60 paid carers, 51 residents with dementia 
(Livingston et al., 2019) and 15 family carers. We anticipated this sample size would provide sufficient information to address our objectives.

\section{Analysis}

\section{Quantitative}

We described participant flow using a consort-type diagram, the number of intervention sessions attended by staff and the proportion of staff attending at least three sessions. We summarised staff, family carer and resident characteristics and questionnaire scores at baseline and follow-up using means with standard deviations (SD) or medians with interquartile ranges (IQR) for continuous data (as appropriate) and frequency (\%) for categorical data. We tabulated $\mathrm{NH}$ characteristics. We compared baseline and follow-up questionnaire scores to obtain an estimate of average change (mean difference) with $95 \%$ confidence intervals. We described missing data for each outcome. Analysis was conducted using Stata version 14 (StataCorp, 2015).

\section{Cost estimation}

We estimated health and social care costs; the intervention cost (including costs of training and delivery) was based on the number and length of sessions delivered and unit costs using published sources (Curtis and Burns, 2017). We explored the feasibility and implications of using the EQ-5D-5L proxy version, DEMQOL proxy version and QUALID to calculate QoL.

\section{Qualitative}

We coded transcripts into meaningful themes using thematic analysis (Braun and Clarke, 2006). We adopted a rigorous approach to data analysis using methods described by Spencer et al. (2003). Throughout the analytic process, researchers used ongoing reflection with the use of memoing and reflective diaries to engage with the data and refine emergent themes.

\section{RESULTS}

\section{Recruitment and consent}

We approached $12 \mathrm{NHs}$, six responded and three agreed to participate. Reasons for $\mathrm{NHs}$ not wishing to participate were having too many other challenges or believing they did not have enough residents with dementia. Recruited NHs varied from 22-215 beds. Two were 
private and one was a charity. At baseline all were rated by the CQC as "good" and had provided staff with dementia training in the prior 6 months (table 1).

A total of 72 staff were working in $\mathrm{NH}$ dementia units and were eligible to participate, 58 of whom were approached by research assistants for informed consent. Fourteen staff were uncontactable by research assistants due to absence, leave or their shift patterns, leading to $74 \%$ of eligible staff approached consented and received the intervention.

Across the three NHs, 57 residents were eligible, 45 had contactable proxies and were approached and 28 proxies provided assent for the resident to participate (49\%). Thus, over half of eligible proxies were not recruited as they either did not respond to the invitation or declined. We approached 28 family carers of residents participating in the project and 13 $(46 \%)$ were recruited into the study. We were able to collect post-intervention questionnaires on 26 residents, 44 staff and 6 family carers (figure 2 ).

\section{Figure 2}

\section{Participant characteristics}

Staff were predominantly female with mean age of 46 years, less than half spoke English as a first language and $19 \%$ had a UK recognised nursing qualification. Residents had a mean age of 87 years and were mainly female. Family carers had a mean age of 62 years and were mainly children of the residents (table 2).

\section{Feasibility}

Staff attended a median of 4 sessions including catch up sessions where necessary (interquartile range (IQR) 3,4), 79\% of staff attended three or four group intervention sessions and including catch up sessions this rose to $84 \%$ of staff (table 1 ).

\section{Potential outcome measures}

Table 3 presents potential outcome measures for a main trial with baseline and follow-up average scores and the numbers who completed these. CMAI, QUALID, SMEOLD, DEMQOL proxy and EQ5-D were completed for at least $89 \%$ of residents at baseline and follow-up. The PAINAD at rest was completed for $78 \%$ of residents at baseline and $73 \%$ at follow-up. Completion of the PAINAD at movement was lower. SWCEOLD data was collected from $92 \%$ of family carers at baseline but only $46 \%$ at follow-up. At baseline the SCID was completed by $94 \%$ of the 53 recruited staff, and all 44 staff who were contactable 
for follow-up. The SCID, SMEOLD and CMAI showed evidence of a positive change between baseline and follow-up.

Pre-intervention on the QUIS, over 10 hours and 53 minutes, we observed 174 interactions with 27 residents. There was a median of 5 (IQR 3,12) interactions per resident, of which 7\% were negative, $51 \%$ neutral and $42 \%$ positive. Post-intervention, over 12 hours and 40 minutes, we observed 126 interactions with 24 residents. There was a median of five interactions per resident (IQR 3, 8), of which $8 \%$ were negative, $46 \%$ neutral and $46 \%$ positive.

\section{Feasibility of cost estimation}

All residents pre-intervention and $93 \%$ of residents post-intervention had complete data for health and social care resource use. Pre-intervention, all residents had complete data for EQ-5D-5L proxy and QUALID and 96\% on DEMQOL proxy. At follow-up, 93\% of the residents had complete data for EQ-5D-5L proxy and QUALID. The total cost of intervention implementation was $£ 6,891.9$. The average cost per resident was $£ 246$, assuming this is the cost required to deliver the intervention to the 28 residents on whom we collected outcome data. There was a slight increase in health and social care costs and QoL measured by DEMQOL proxy and QUALID, and a statistically significant increase in QoL measured by EQ-5D-5L proxy (see appendix 1 published as supplementary material online attached to the electronic version of this paper at https://www.cambridge.org/core/journals/internationalpsychogeriatrics).

\section{Qualitative evaluation}

We interviewed ten family carers in nine interviews (one with two family carers). All were women (nine daughters, one wife and one niece), aged 50-73 years who visited between seven and 30 times per month. We held five staff focus groups, two in two of the $\mathrm{NHs}$, and one in the other. Staff members (17 females, 2 males) attended in groups varying in size from two to seven staff members. There were 13 healthcare assistants, two senior healthcare assistants, one each of assistant nursing practitioner, unit manager, clinical lead, and $\mathrm{NH}$ manager.

\section{Creating a shared learning space and enhancing communication}

Facilitators built rapport with staff who reported feeling listened to, understood and appreciated. The training kept staff engaged and brought those with different roles together 
Improving care for agitation in dementia at end-of-life

to share experiences.

“...they [facilitators] were interested in listening to us... asking us questions..." "...they were giving us new tools, ways of looking at pain, doing things differently... "I liked them very much ...It was their way of teaching, .., it was like communication."(focus group HCA1)

"And it was good that we had nurses and senior healthcare assistants as well [in the training]... they will be more informed... if we were in the sessions and sharing something out, like for instance palliative care, you find that the nurse or the senior healthcare assistant will have different input than we healthcare assistants coming up with. So, it did help to get everybody together. (focus group HCA2)

\section{Investing in staff}

Staff reported the training helped them feel valued, reflect on their work and increased quality of care. The training was relevant to their day-to-day practice and was easy to apply: It was preferred to "learning on the job".

“...it's more practical...for instance... I'm new and haven't been in a dementia facility, and I get introduced to the sessions. I think it would be more beneficial...I'm reflecting back to the sessions. This is what l've learned .., so I am just going to put it into practice...Even though I've been here for three years, it's still beneficial to me, and it would be beneficial for a new person as well,." (HCA2)

“... we reflect back to your training... probably we need to do more training to exercise our minds." (HCA5)

Manuals helped staff to follow the progression of the sessions, to make notes and provided written material for the future. The person-centred approach was valued by carers:

"...we can go back to the manuals, and when we reflect, it's something we can go back to and read, and then, we can reflect back on what we talked about in the training." (HCA2)

"Because they think person-centred means that this person likes trees and they don't like strawberries, you know. I think person centred means looking at somebody in a room and saying why are they agitated or why, what can I do to make their care, make them more comfortable?" (family carer 3 )

Linking sessions through the introduction, recap and between-session tasks helped put it all together. 
Improving care for agitation in dementia at end-of-life

“...from one session to next session, we reflect on the first one, then we carry along. So it's more covered and go round like a ball throughout... We tend to understand better because it's broken down to four sections. If... everything together, probably we would not". (HCA3)

Relaxation techniques were regarded as valuable, enjoyable, and useful for relieving stress.

“...[relaxation exercises] are excellent...staff stress is hugely important...[their work] is immensely difficult and distressing... [staff] cope with enormous amount of stress..." (family carer 1)

"I think the relaxation side...that was the best part. Because I work in the dementia unit, sometimes can be a bit challenging, so I find that very good, using that is helpful." (HCA6)

Family carers felt the training encouraged better communication and interaction between staff and relatives.

"...the training you are doing, is about making something that is often not implicit, but unvoiced, voiced." (Family carer 3)

Family carers judged the training as useful and valued the focus on agitation:

"...how to handle it [agitation], or what to do, or what would help...that is crucial..." "...that's [aggression] is really one of the most difficult things to deal with, for them [staff]...my dad gets very aggressive, and I'm sure they find that very difficult. I think also, if people can't communicate...not knowing what to do and not knowing if things are helping..." (family carer 2)

\section{Culture and implementation in practice}

Staff understood that implementation and change takes time.

“...when you learn something new and you want to put it into practice, it takes a while...You know, people have habits." (HCA1)

A family carer was concerned about the "culture of blame". Staff may hesitate to raise issues, afraid of accountability. The carer felt that the training would help this.

"...if a care home creates a culture where it's everybody's job to think about the person and their comfort level, I think that would translate into better care." (family carer 4) 
There was some concern about training costs but the delivery by non-professional facilitators was appropriate, suitable and implementable.

"If you go for training, they [NH] have to put on more staff which is more money for them...it's time consuming... but I don't think they will say no to it... we are learning something..." (HCA2)

\section{Applying skills in practice}

Many staff put new skills into practice at work, sometimes immediately after the sessions.

"We really put it into production more, is like asking the daughter, did your mum love music? Do you think she preferred to listen to music in the background... We know about these things, but sometimes we don't really put it into action, but after listening to the end-of-life training, then yes, we did." (HCA1).

\section{DISCUSSION}

Using a palliative care approach, underpinned by theory and ethnographic work, we designed a four-session manualised training intervention to improve the care of people with severe dementia and agitation. We recruited our target of three $\mathrm{NHs}, 74 \%$ of eligible staff and $89 \%$ of family carers. We had aimed to recruit 17 residents with severe dementia from each $\mathrm{NH}$ (total of 51 ) but only recruited 28 in total (49\% of eligible residents). Most (84\%) staff attended at least three sessions.

We explored potential outcome measures for a full trial. In terms of feasibility, nearly all data on agitation, symptom management (SMEOLD) and quality of life (DEMQOL, QUALID and EQ-5D-5L) were collected although other scales including the PAINAD, particularly at movement were less consistently measured.

We wanted to explore the quality of interactions between $\mathrm{NH}$ residents and staff. We chose the QUIS as previous studies show utility in NHs (Proctor et al., 1998) and with cognitively impaired NH residents (Paudel et al., 2019). We were able to assess daytime resident-staff interactions for $96 \%$ of residents at baseline and $92 \%$ at follow-up. Thus, it was feasible to observe staff interactions with residents with severe dementia. Dementia Care Mapping is used more frequently to assess person-centred care but requires extensive training, which may be less practical. The median number of interactions and the type of interaction did not change between baseline and follow-up. 
Improving care for agitation in dementia at end-of-life

The SCID (staff competency) scale and the symptom management scale (SMEOLD) showed positive change between pre and post-intervention phases, indicating they may be useful outcome measures for a future study. It was feasible to estimate intervention costs using DEMQOL proxy and the EQ-5D-5L, suggesting a potential cost of £246 per resident. Health and social care costs and utility values showed slight increase post-intervention.

Analysis of qualitative data allowed triangulation with quantitative findings. The training was acceptable to staff who appreciated multi-professional learning, interactions with facilitators who listened to them, the ability to put their learning quickly into practice and the focus on their wellbeing and personhood through reflection and relaxation exercises.

There are a number of limitations to this feasibility study. The NHs in which the study was conducted may be atypical. Participating NHs were rated as "good" by the regulator and thus likely to be more willing and able to participate in research; they may not be representative. We did not formally assess how well $\mathrm{NH}$ managers engaged with the intervention and their support is vital for implementation (Chenoweth et al., 2018) We recruited a relatively high proportion of eligible staff, but did not recruit residents or their carers to target. Relatively few residents fulfilled the criteria, despite these being quite broad and crucially, few had proxies who could participate to allow collection of family carer data. It may be challenging to recruit enough dyads for a full-scale trial. We did not collect data on the level of intervention fidelity achieved by the facilitators. Focus group participants may have been wary of criticising the intervention, however, we did ensure these occurred after the intervention finished enabling time for reflection, and they were conducted by researchers who did not deliver the intervention. Cost estimates are conservative and interpreted with caution because of small sample size and lack of control arm.

This feasibility study informs future work in this area. We did not aim to detect differences pre or post-intervention, but found signals of improvement in staff competency (SCID scale) and resident symptom management (SMEOLD). More work is required to understand whether these are true changes, underlying mechanisms of change and the "active ingredient" of the intervention. Whilst improving factual knowledge is important, it may be that engagement of the facilitators and attention given to staff wellbeing and personhood were actually the mediators of change. Future research should assess staff wellbeing, exploring emotional distress, anxiety and job satisfaction. We do not have data on longerterm implications of the study including lasting culture change within the NHs.

There has been an increase in recent research on interventions to improve end-of-life care for $\mathrm{NH}$ residents, particularly those with dementia. Many of these interventions are complex, 
for example the UK "Gold Standards Framework" (Gold Standards Framework, 2008) or the Palliative Care for Older People (PACE) Steps to Success Program, a multicomponent intervention aiming to integrate basic non-specialist palliative care in $\mathrm{NHs}$ (Van den Block et al., 2019). Whilst palliative care takes a broad and holistic approach, it is challenging for complex interventions to achieve their desired outcomes such as improved resident comfort or staff knowledge (Van den Block et al., 2019).

$\mathrm{NH}$ residents with severe dementia have complex needs requiring specialised support from external health and social care services (Sampson 2018). Many multicomponent interventions involve expensive external facilitation from senior staff, training and multidisciplinary input. It may be more feasible in the current climate to implement focused interventions on agitation, advance care planning (Livingston et al., 2013) or pain management (Petyaeva et al., 2018). However, we still lack evidence for the effectiveness of these approaches (Murphy et al., 2016).

\section{Conclusion}

This is the first feasibility study of an intervention designed to improve agitation in $\mathrm{NH}$ residents with severe dementia underpinned by a palliative care approach. The training was acceptable, positively received by staff and family carers, and inexpensive. Further studies will be required to understand implementation and how the intervention promotes change in practice.

\section{Conflict of interest declaration: none}

Description of authors' roles: GL \& ELS obtained funding, designed the study and protocol. LM, JB, MP designed and conducted the quantitative data analyses. AL, AS, FLaF, $\mathrm{KL}$, JG, LW, MM, KM were involved in intervention design, delivery, data acquisition and analysis. AL was study manager. All authors were involved in data interpretation and drafting the paper.

Acknowledgements: Funded by the UK Economic and Social Research Council and National Institute of Health Research (NIHR/ESRC ES/L001780/1). ELS is supported by Marie Curie core grant (grant number MCCC-FCO-16-U). GL and ELS are supported by National Institute for Health Research (NIHR) University College London Hospital Biomedical Research Centre. GL received funding from the NIHR Collaborations for Leadership in Applied Health Research and Care North Thames at Barts Health National Health Service (NHS) Trust. GL is an NIHR senior investigator. Views expressed are those of the authors and not necessarily those of the NHS, the NIHR, or the UK Department of Health. We thank all participating NHs, residents, families, and staff. 
Improving care for agitation in dementia at end-of-life

\section{REFERENCES}

Aminoff, B. Z. and Adunsky, A. (2006). Their last 6 months: suffering and survival of endstage dementia patients. Age Ageing, 35, 597-601.

Beecham, J. and Knapp, M. (2001). Costing psychiatric interventions. In G. Thornicroft (Ed.) Measuring Mental Helath Needs (pp. 200-224) London: Gaskell.

Braun, V. and Clarke, V. (2006). Using thematic analysis in psychology. Qualitative Research in Psychology, 3, 24.

Chenoweth, L., Jessop, T., Harrison, F., Cations, M., Cook, J. and Brodaty, H. (2018). Critical Contextual Elements in Facilitating and Achieving Success with a Person-Centred Care Intervention to Support Antipsychotic Deprescribing for Older People in Long-Term Care. Biomed Res Int, 2018, 7148515.

Clark, D. (1999). 'Total pain', disciplinary power and the body in the work of Cicely Saunders, 1958-1967. Soc Sci Med, 49, 727-736.

Cohen-Mansfield, J., Marx, M. S. and Rosenthal, A. S. (1989). A description of agitation in a nursing home. J.Gerontol., 44, M77-M84.

Curtis, L. and Burns, A. (2017). Unit Costs of Health and Social Care 2017. Personal Social Services Research Unit, University of Kent, Canterbury. .

Dean, R., Proudfoot, R. and Lindesay, R. (1993). The quality of interactions schedule (QUIS): development, realiability amd use in the evaulation of two domus units. International Journal of Geriatric Psychiatry, 8, 7.

Devlin, N. J., Shah, K. K., Feng, Y., Mulhern, B. and van Hout, B. (2017). Valuing healthrelated quality of life: An EQ-5D-5L value set for England. Health Econ.

Froggatt, K. A., et al. (2006). End-of-life care in long-term care settings for older people: a literature review. Int J Older People Nurs, 1, 45-50.

Gold Standards Framework (2008). The Gold Standards Framework. NHS End of Life Care Programme.

Herdman, M., et al. (2011). Development and preliminary testing of the new five-level version of EQ-5D (EQ-5D-5L). Qual Life Res, 20, 1727-1736.

Husebo, B. S., Ballard, C., Sandvik, R., Nilsen, O. B. and Aarsland, D. (2011). Efficacy of treating pain to reduce behavioural disturbances in residents of nursing homes with dementia: cluster randomised clinical trial. BMJ, 343, d4065.

Kadri, A., Rapaport, P., Livingston, G., Cooper, C., Robertson, S. and Higgs, P. (2018). Care workers, the unacknowledged persons in person-centred care: A secondary qualitative analysis of UK care home staff interviews. PloS one, 13, e0200031.

Kiely, D. K., Volicer, L., Teno, J., Jones, R. N., Prigerson, H. G. and Mitchell, S. L. (2006). The validity and reliability of scales for the evaluation of end-of-life care in advanced dementia. Alzheimer Dis.Assoc.Disord., 20, 176-181.

Laing and Buisson (2017). Care of Older People UK Market Report.

Levin, E., Sinclair, I. and Gorbach, P. (1989). Families, services, and confusion in old age. Aldershot: Avebury: National Institute for Social Work.

Livingston, G., et al. (2017). Prevalence of and associations with agitation in residents with dementia living in care homes: MARQUE cross-sectional study. BJPsych Open, 3, 171-178. Livingston, G., et al. (2019). Clinical and cost-effectiveness of the Managing Agitation and Raising Quality of Life (MARQUE) intervention for agitation in people with dementia in care homes: a single-blind, cluster-randomised controlled trial. Lancet Psychiatry, 6, 293-304.

Livingston, G., et al. (2014a). Non-pharmacological interventions for agitation in dementia: systematic review of randomised controlled trials. Br J Psychiatry, 205, 436-442.

Livingston, G., et al. (2014b). A systematic review of the clinical effectiveness and costeffectiveness of sensory, psychological and behavioural interventions for managing agitation in older adults with dementia. Health Technol Assess, 18, 1-226, v-vi.

Livingston, G., et al. (2013). Improving the end-of-life for people with dementia living in a care home: an intervention study. Int Psychogeriatr, 25, 1849-1858. 
McLean, C., Griffiths, P., Mesa-Eguiagaray, I., Pickering, R. M. and Bridges, J. (2017). Reliability, feasibility, and validity of the quality of interactions schedule (QuIS) in acute hospital care: an observational study. BMC Health Serv Res, 17, 380.

Mitchell, S. L., et al. (2009). The clinical course of advanced dementia. N.Engl.J.Med., 361, 1529-1538.

Moore, K. J., et al. (2017a). Implementing the compassion intervention, a model for integrated care for people with advanced dementia towards the end of life in nursing homes: a naturalistic feasibility study. BMJ Open, 7, e015515.

Moore, K. J., et al. (2017b). Experiences of end of life amongst family carers of people with advanced dementia: longitudinal cohort study with mixed methods. BMC Geriatr, 17, 135.

Moriarty, J. and Webb, S. (2000). Part of their lives: community care for older people with dementia. Bristol. Bristol: Policy Press.

Morris, J. C. (1993). The Clinical Dementia Rating (CDR): current version and scoring rules. Neurology, 43, 2412-2414.

Murphy, E., et al. (2016). Palliative care interventions in advanced dementia. Cochrane Database Syst Rev, 12, CD011513.

Orellana, K. (2014). Care Home Managers: A scoping review of evidence. London: Kings College.

Paudel, A., Resnick, B. and Galik, E. (2019). The Quality of Interactions Between Staff and Residents With Cognitive Impairment in Nursing Homes. Am J Alzheimers Dis Other Demen, 1533317519863259.

Petyaeva, A., et al. (2018). Feasibility of a staff training and support programme to improve pain assessment and management in people with dementia living in care homes. Int $J$ Geriatr Psychiatry, 33, 221-231.

Prince, M., Wimo, A. G. M., Ali, G. C., Wu, Y. T. and Prina, M. (2015). World Alzheimer Report 2015 - The Global Impact of Dementia: An Analysis of Prevalence, Incidence, Cost and Trends. London.

Proctor, R., et al. (1998). An observational study to evaluate the impact of a specialist outreach team on the quality of care in nursing and residential homes. Aging and Mental Health, 2, 6.

Rabin, R. and de, C. F. (2001). EQ-5D: a measure of health status from the EuroQol Group. Ann.Med., 33, 337-343.

Rapaport, P., et al. (2018). How do care home staff understand, manage and respond to agitation in people with dementia? A qualitative study. BMJ Open, 8, e022260.

Sampson, E. L., et al. (2018). Living and dying with advanced dementia: A prospective cohort study of symptoms, service use and care at the end of life. Palliat Med, 32, 668-681.

Sampson, E. L., et al. (2019). Agitation near the end of life with dementia: An ethnographic study of care. PloS one, 14, e0224043.

Schepers, A. K., Orrell, M., Shanahan, N. and Spector, A. (2012). Sense of competence in dementia care staff (SCIDS) scale: development, reliability, and validity.

Int.Psychogeriatr., 24, 1153-1162.

Skills for Care (2019). National Minimum Dataset for Social care United Kingdom.

Sleeman, K. E., Davies, J. M., Verne, J., Gao, W. and Higginson, I. J. (2016). The changing demographics of inpatient hospice death: Population-based cross-sectional study in England, 1993-2012. Palliat Med, 30, 45-53.

Smets, T., et al. (2018). Integrating palliative care in long-term care facilities across Europe (PACE): protocol of a cluster randomized controlled trial of the 'PACE Steps to Success' intervention in seven countries. BMC Palliat Care, 17, 47.

Smith, S. C., et al. (2007). Development of a new measure of health-related quality of life for people with dementia: DEMQOL. Psychol Med, 37, 737-746.

Spencer, L., Ritchie, J., Lewis, J. and Dillon, L. (2003). Quality in qualitative evaluation: A framework for assessing research evidence.

StataCorp (2015). Stata Statistical Software: Release 14. College Station, TX StataCorp LP. 
Van den Block, L., et al. (2019). Evaluation of a Palliative Care Program for Nursing Homes in 7 Countries: The PACE Cluster-Randomized Clinical Trial. JAMA Intern Med, 1-10. van der Steen, J. T. and Goodman, C. (2015). What research we no longer need in neurodegenerative disease at the end of life: the case of research in dementia. Palliat Med, 29, 189-192.

van der Steen, J. T., et al. (2014). White paper defining optimal palliative care in older people with dementia: a Delphi study and recommendations from the European Association for Palliative Care. Palliat Med, 28, 197-209.

Warden, V., Hurley, A. C. and Volicer, L. (2003). Development and psychometric evaluation of the Pain Assessment in Advanced Dementia (PAINAD) scale.

J.Am.Med.Dir.Assoc., 4, 9-15.

Weiner, M. F., Martin-Cook, K., Svetlik, D. A., Saine, K., Foster, B. and Fontaine, C. S. (2000). The quality of life in late-stage dementia (QUALID) scale. J.Am.Med.Dir.Assoc., 1, 114-116.

World Health Organisation (1990). Cancer Pain Relief and Palliative Care. Geneva.

World Health Organisation (2017). Global Health Observatory (GHO) data; mortality and global health estimates.

Xie, J., Brayne, C. and Matthews, F. E. (2008). Survival times in people with dementia: analysis from population based cohort study with 14 year follow-up. BMJ, 336, 258-262.

\section{FIGURE LEGENDS}

Figure 1. Intervention content and delivery

Figure 2. Recruitment flow chart

\section{APPENDIX}

Appendix 1: Mean health and social care resource use and cost per resident at baseline and follow-up and mean total cost including intervention

(appendix 1 published as supplementary material online attached to the electronic version of this paper at https://www.cambridge.org/core/journals/international-psychogeriatrics) 
Table 1: Characteristics of participating nursing homes

\begin{tabular}{|c|c|c|c|}
\hline & Site 1 & Site 2 & Site 3 \\
\hline \multicolumn{4}{|l|}{ Ownership and registration } \\
\hline Ownership & Charity & Private & Private \\
\hline Dementia specialist & $\mathrm{N}$ & $\mathrm{Y}$ & $\mathrm{Y}$ \\
\hline Mental health registered & $\mathrm{N}$ & $\mathrm{Y}$ & $\mathrm{N}$ \\
\hline Physical disability registered & $\mathrm{N}$ & $\mathrm{Y}$ & $\mathrm{Y}$ \\
\hline CQC rating - overall & Good & Good & Good \\
\hline \multicolumn{4}{|l|}{ Nursing home size and type of residents } \\
\hline Number of beds & 215 & 22 & 52 \\
\hline Number of residents present in home & 184 & 22 & 51 \\
\hline Number of residents with dementia in the home & 110 & 22 & 25 \\
\hline Number of residents in hospital & 1 & 0 & 1 \\
\hline New residents with dementia are cared for in a specialist area & $\mathrm{Y}$ & $\mathrm{N}$ & $\bar{Y}$ \\
\hline Special unit for residents with dementia and behavioural disturbances & $\bar{Y}$ & $\mathrm{~N}$ & $\bar{N}$ \\
\hline Home has a specific team for dementia care & $\mathrm{Y}$ & $\mathrm{Y}$ & Y \\
\hline Residents are moved as their needs change & $\mathrm{Y}$ & $\mathrm{N}$ & $\bar{N}$ \\
\hline Number of nursing staff rostered on during the day in the last 24 hours & 3 & 1 & 2 \\
\hline Number of care staff rostered on during the day in the last 24 hours & 36 & 5 & 10 \\
\hline Number of nursing staff rostered at night in the last 24 hours & 3 & 1 & 2 \\
\hline Number of care staff rostered on during the night in the last 24 hours & 36 & 2 & 4 \\
\hline Number of staff in the last 24 hours who were agency or bank & 8 & 0 & 0 \\
\hline Number of permanently registered nursing staff & 14 & 6 & 11 \\
\hline Number of permanently registered care staff & 168 & 17 & 44 \\
\hline Number of permanently registered nursing staff on leave & - & 1 & 1 \\
\hline Number of permanently registered care staff on leave & - & 0 & 0 \\
\hline $\begin{array}{l}\text { Number of registered nurses joined as permanent staff in last } 4 \\
\text { months }\end{array}$ & 0 & 0 & 3 \\
\hline Number of other care staff joined as permanent staff in last 4 months & 4 & 4 & 3 \\
\hline Nursing home provided dementia training in the last six months & $\mathrm{Y}$ & $\mathrm{Y}$ & $\bar{Y}$ \\
\hline \multicolumn{4}{|l|}{ Recruitment at baseline } \\
\hline Residents & 14 & 7 & 7 \\
\hline Staff & 29 & 12 & 12 \\
\hline Family carers & 5 & 2 & 6 \\
\hline \multicolumn{4}{|l|}{ Training delivered } \\
\hline Number of intervention training groups in the care home & 4 & 2 & 2 \\
\hline
\end{tabular}

Table 1 legend: $Y=$ yes, $N=$ no 
Table 2: Characteristics of participants

\begin{tabular}{|c|c|}
\hline Characteristic & $\begin{array}{r}\text { Frequency/N (\%) } \\
\text { unless stated otherwise }\end{array}$ \\
\hline \multicolumn{2}{|l|}{ STAFF $(\mathrm{N}=53)$} \\
\hline Female & $48 / 53(91 \%)$ \\
\hline Age mean (SD) & $46(14)$ \\
\hline \multicolumn{2}{|l|}{ Ethnicity $(\mathrm{N}, \%)$} \\
\hline White & $5 / 52(10 \%)$ \\
\hline Black & $26 / 52(50 \%)$ \\
\hline Asian & $14 / 52(27 \%)$ \\
\hline Mixed or other & $7 / 52(13 \%)$ \\
\hline \multicolumn{2}{|l|}{ Educational attainment } \\
\hline No qualifications/ O levels/ GCSEs/ CSEs/ NVQ/ Level 1 or 2 & $14 / 53(26 \%)$ \\
\hline A levels/ NVQ Level 3-5 & $15 / 53(28 \%)$ \\
\hline Degree/ Postgraduate & $19 / 53(36 \%)$ \\
\hline Other & $5 / 53(9 \%)$ \\
\hline English is first language & $23 / 53(43 \%)$ \\
\hline Years working in any nursing home mean (SD) & $6(6)$ \\
\hline Years working in current nursing home mean (SD) & $3(4)$ \\
\hline Full time work $(\mathrm{N}, \%)$ & $47 / 53(89 \%)$ \\
\hline Part time work (N, \%) & $6 / 53(11 \%)$ \\
\hline \multicolumn{2}{|l|}{ Shift pattern (N, \%) } \\
\hline Days & $38 / 52(73 \%)$ \\
\hline Days and nights & $14 / 52(27 \%)$ \\
\hline UK recognised nursing qualification & $10 / 52(19 \%)$ \\
\hline Registered general nurse & $7 / 10(70 \%)$ \\
\hline Registered mental health nurse & $2 / 10(20 \%)$ \\
\hline Enrolled nurse & $1 / 10(10 \%)$ \\
\hline \multicolumn{2}{|l|}{ RESIDENTS ( $\mathrm{N}=28)$} \\
\hline Female Residents & $19 / 28(68 \%)$ \\
\hline Age mean (SD) (N=27) & $87(9)$ \\
\hline \multicolumn{2}{|l|}{ Ethnicity } \\
\hline White & $26 / 27(96 \%)$ \\
\hline Non-white & $1 / 27(4 \%)$ \\
\hline \multicolumn{2}{|l|}{ Educational attainment } \\
\hline No qualifications & $2 / 15(13 \%)$ \\
\hline Degree/ postgraduate & $11 / 15(73 \%)$ \\
\hline Other & $2 / 15(13 \%)$ \\
\hline English is first language & $19 / 23(83 \%)$ \\
\hline \multicolumn{2}{|l|}{ Marital status } \\
\hline Married & $12 / 27(44 \%)$ \\
\hline Separated/ divorced & $3 / 27(11 \%)$ \\
\hline Widow/ widower & $12 / 27(44 \%)$ \\
\hline \multicolumn{2}{|l|}{ FAMILY CARERS $(\mathrm{N}=13)^{*}$} \\
\hline Female & $10 / 11(91 \%)$ \\
\hline Age mean (SD) & $62(7)$ \\
\hline \multicolumn{2}{|l|}{ Relationship to resident } \\
\hline Spouse & $3 / 11(27 \%)$ \\
\hline Child & $7 / 11(64 \%)$ \\
\hline Other & $1 / 11(9 \%)$ \\
\hline Visits per month median (IQR) & $13(7,22)$ \\
\hline
\end{tabular}

IQR - interquartile range. $S D=$ Standard deviation

*2 family carers did not provide demographic information 
Table 3: Study measures

\begin{tabular}{|c|c|c|c|c|c|c|c|}
\hline & \multicolumn{2}{|r|}{ Baseline } & \multicolumn{2}{|r|}{ Follow up } & \multicolumn{3}{|c|}{ Difference (Follow up - baseline) } \\
\hline & $\mathbf{N}$ & Mean (SD) & $\mathbf{N}$ & Mean (SD) & $\mathbf{N}$ & Mean difference & $\begin{array}{l}95 \% \text { Confidence } \\
\text { interval }\end{array}$ \\
\hline STAFF SCIDS & 53 & & 44 & & & & \\
\hline Professionalism & 51 & $18(2)$ & 44 & $19(1)$ & 43 & 0.3 & $(-0.2,0.9)$ \\
\hline Building Relationships & 53 & $13(2)$ & 44 & $14(2)$ & 44 & 0.7 & $(0.1,1.3)$ \\
\hline Care Challenges & 52 & $14(2)$ & 44 & $15(2)$ & 43 & 0.8 & $(0.2,1.4)$ \\
\hline Sustaining Personhood & 52 & $15(1)$ & 44 & $15(1)$ & 43 & 0.5 & $(-0.0,1.0)$ \\
\hline Overall & 50 & $60(6)$ & 44 & $63(5)$ & 42 & 2.4 & $(0.5,4.2)$ \\
\hline RESIDENTS & 28 & & 26 & & & & \\
\hline $\mathrm{CMAl}^{*}$ & 23 & $\begin{array}{c}44(20) \\
\text { Median (IQR): } \\
34(30,57)\end{array}$ & 26 & $\begin{array}{c}42(13) \\
\text { Median (IQR): } \\
37(31,52)\end{array}$ & 21 & $\begin{array}{c}-4.9 \\
\text { Median difference: }-2\end{array}$ & $\begin{array}{c}(-10.4,0.7) \\
(-8,0)\end{array}$ \\
\hline PAIN-AD at rest & 22 & $1(4)$ & 19 & $1(2)$ & 21 & -0.2 & $(-2.3,2.0)$ \\
\hline PAIN-AD at movement & 19 & $1(2)$ & 19 & $1(1)$ & 15 & 0.1 & $(-0.9,1.2)$ \\
\hline Quality of life in late stage dementia (QUALID) & 27 & $22(7)$ & 26 & $23(8)$ & 25 & 0.9 & $(-2.7,4.4)$ \\
\hline SMEOLD & 27 & $27(10)$ & 26 & $33(8)$ & 25 & 5.7 & $(1.4,9.9)$ \\
\hline DEMQOL proxy & 28 & $107(12)$ & 26 & $113(9)$ & 26 & 4.5 & $(0.0,9.1)$ \\
\hline FAMILY CARERS & 13 & & 6 & & & & \\
\hline Carer SWCEOLD & 12 & $29(3)$ & 6 & $28(4)$ & 6 & -1.7 & $(-5.3,1.9)$ \\
\hline
\end{tabular}

$\mathrm{SD}=$ standard deviation, $\mathrm{IQR}=$ interquartile range, ${ }^{*}$ median and median difference also given as the score has a skewed distribution 